\title{
Evolution of Lung Cancer Chemotherapy: A Brief Review
}

\author{
Silpa Kshetrimayum
}

\begin{abstract}
Lung cancer is one of the most lethal forms of cancer worldwide. Even in this era of advances in cancer treatment modalities, the mortality of lung cancer continues to rise. Lung cancer is notorious for detection usually in an advanced stage where surgery has very little role. Targeted therapy in the form of various classes of tyrosine kinase inhibitors offer a great option for better lung cancer management.
\end{abstract}

Keywords: cancer, lung, targeted, chemotherapy, tyrosine-kinase

Once thought to be among the rarest forms of cancer, lung cancer is now one of the commonest cancers in the world and is also the leading cause of cancer associated deaths among both men and women worldwide[1]. The first half of the twentieth century saw an enormous increase in the number of lung cancer cases almost in concordance with the growing cigarette smoking epidemic. Although the incidence of lung cancer is showing a declining trend in the developed countries, there has been a relative increase in the developing countries. Attributable to the increase in global tobacco use, especially in Asia, the WHO estimates that lung cancer mortality will continue to rise worldwide[2].

Despite newer advances in surgery and chemotherapy, the prognosis of lung cancer remains grim with an overall 5 year survival of approximately $15 \%$ in the developed and $5 \%$ in the developing countries[3]. Like all other cancers, lung cancer is the cumulative result of a number of genetic changes in the bronchial epithelial cells brought over time by a number of carcinogenic agents. These genetic and various epigenetic mechanisms interplay to ultimately result in the cancer phenotype. Though these molecular changes are complex, the past decades have seen the discovery of some specific mutations responsible for the malignant phenotype known as 'driver mutations'. The recognition of these mutations and the possibility of designing an inhibitor for the same have opened up enormous prospects for cancer chemotherapy.

More than $70 \%$ lung cancer patients present in the advanced stage when the tumour is practically unresectable [4]. Chemotherapy is therefore the main focus for newer research today. Histologically, lung cancer is broadly classified into small cell lung carcinoma (SCLC) and non small cell lung cancer (NSCLC) which is further classified into large cell carcinoma, adenocarcinoma and squamous cell carcinoma[5]. Earlier chemotherapy regimens were decided based on this classification. A cisplatin-etoposide regimen with or without thoracic and prophylactic cranial irradiation is the standard care for advanced SCLC[6]. The distinction of NSCLC into adenocarcinoma and squamous cell type was of little relevance at that time as the same combination chemotherapy of a platinum compound with gemcitabine was administered in both cases. Pemetrexed, an anti-folate agent got FDA approval in 2004 for use as second line therapy in patients with advanced NSCLC [7]. Based on the results of a phase III trial of first line pemetrexed, this recommendation was changed to use only in patients with non squamous histology in 2008[8].

With the advent of 'Genomics' and the discovery of a number of molecular abnormalities and mutations in driver genes especially EGFR, KRAS, BRAF, HER2, AKT1, NRAS, PIK3CA, MEK1, EML4-ALK, MET, MYC[9], etc., therapeutic options have greatly increased. Histologic classification of lung cancer is now extending to molecular classification. These mutation sites serve as therapeutic 'targets' which lead to specific lethality for only tumour cells and spare normal cells. Driver mutations in the epidermal growth factor receptor (EGFR) gene, a membrane bound tyrosine kinase, was discovered in NSCLC, mainly adenocarcinoma, in 2004. It was found that the mutations occurred exclusively in the TK region, that is, exon 18 to 24. First generation TKIs namely Gefitinib and Erlotinib were developed shortly after and various studies were conducted to compare their efficacy against conventional chemotherapy. CALGB 30406[10], a study among Caucasians and IPASS[11] and FIRST SIGNAL[12], studies among East Asians showed that EGFR TKI as first line chemotherapy for advanced NSCLC was not inferior to standard chemotherapy in terms of objective response rate (ORR), progression free survival (PFS) and overall survival (OS) . The patients in these studies were selected on the basis of the earlier observation that patients who were females, Asians, non-smokers and had adenocarcinoma histology responded better to EGFR-TKIs. It is now recommended that all patients with adenocarcinoma histology be evaluated for EGFR mutation. EGFR-TKIs are now recommended as first line treatment in advanced EGFR mutation positive adenocarcinoma[13]. The prevalence of EGFR mutation in adenocarcinoma is about $40 \%$ in East Asians and $15 \%$ in Caucasians[14]. Thus EGFR TKIs have the potential to benefit a good number of patients with lung cancer.

The main limitation of EGFR-TKI is the development of resistance. All patients who initially respond to EGFR inhibitors ultimately relapse. Two mechanisms have been proposed for this 'acquired' resistance. First is the appearance of a single point mutation in the 'gatekeeper' gene located alongside the EGFR gene in exon 20. This mutation (T790M) causes conformational change in the EGFR receptor thereby preventing the binding of TKI to the EGFR[15]. The second mechanism is MET oncogene amplification which causes activation of downstream 


\section{International Journal of Science and Research (IJSR) \\ ISSN (Online): 2319-7064}

Index Copernicus Value (2015): 78.96 | Impact Factor (2015): 6.391

pathway in the absence of activation of the EGFR-TK due to binding with inhibitor [14]. Thus the need for a novel drug that would work on gefitinib and erlotinib resistant tumours gained profound importance.

Afatinib is a second generation irreversible inhibitor of receptor tyrosine kinase that has activity on all receptors of the ERBB family (EGFR, HER2, ERBB3 and ERBB4). It binds covalently to these receptors and blocks signaling irreversibly. Afatinib has also been shown to have activity on receptors with the resistance conferring T790M mutation[16]. The LUX lung clinical trial programme was launched in 2011 to investigate the use of afatinib in different settings of advanced NSCLC (LUX lung 1, 2, 3, 4, $5,6,7,8)$. Most of the studies showed a favourable secondary outcome in terms of longer progression free survival with afatinib compared to platinum doublet chemotherapy when used as first or second line therapy. Overall survival (primary outcome) has been assessed from two randomized, phase 3 trials, LUX lung 3 and LUX lung 6. The analysis has shown that there is definite overall survival benefit in patients with tumours harbouring the EGFRdel19 mutation but not those with L858R positive mutations compared to cisplatin based doublet chemotherapy[17]. The early results of LUX lung 7, a phase IIb trial directly comparing gefitinib with afatinib has shown superior efficacy of afatinib. Risk of lung cancer was reduced by $27 \%$ in the afatinib group compared to gefitinib (PFS 10.1 months against 8.4 months). The primary outcome analysis report of overall survival (OS) is awaited[18]. Another second generation TKI under trial is PF299804 (Dacomitinib). It is a pan-HER inhibitor. The results of a phase II trial had been promising [19] but the ARCHER 1009 trial comparing it with erlotinib failed to meet its objective of prolonging overall survival (OS) and the drug has gone into disfavor. Third generation TKIs undergoing trials are CO-1686 [rociletinib], AZ9291, HM61713, EGF816, and ASP8273. They preferentially inhibit EGFR T790M but have no activity on the wild type EGFR [20].

EGFR TKIs are remarkably well tolerated. Typical side effects include dermatological toxicities like rash, acne and pruritis and diarrhea. Liver toxicity has been reported but it is usually reversible. Interstitial lung disease was seen in 1$3 \%$ patients in some studies [14].

Targeted therapy has thus opened the door to immense possibilities for drug therapy in lung cancer. Targeted therapy along with preventive measures like smoking cessation programmes promise to serve as the most effective tools in the battle against lung cancer.

\section{References}

[1] Ferlay J, Soerjomataram I, Ervik M, Dikshit R, Eser S, Mathers C, et al. GLOBOCAN 2012 v1.0, Cancer Incidence and Mortality Worldwide: IARC CancerBase No. 11. Lyon, France: International Agency for Research on Cancer; 2013

[2] Parkin DM, Bray F, Ferlay J, Pisani P. Global cancer statistics, 2002. CA Cancer J Clin 2005; 55 : 74-108.
[3] Jemal A, Bray F, Center MM, Ferlay J, Ward E, Forman D. Global Cancer Statistics. CA Cancer J Clin . 2011;61 (2):69-90.

[4] William D, Travis. Pathology of lung cancer. Clinics in Chest Medicine, Saunders Dec 2011;32(4):669.

[5] Travis W, Brambila E, et al. International association for the study of lung cancer/American Thoracic Society/European Respiratory Society International Multidisciplinary Classification of Lung Adenocarcinoma.J Thorac Oncol. 2011;6: 244-285

[6] Dowell J, gerber D, Johnson D. Small cell lung cancer: diagnosis, treatment, and natural history. Fishman's pulmonary diseases and disorders. $5^{\text {th }}$ ed. USA: Mc Graw Hill, 2015: 1770-1781.

[7] Hanna N, Shepherd FA, Fosella FV, et al. Randomised Phase III trial of pemetrexed versus docetaxel in patients with non small cell lung cancer previously treated with chemotherapy. J Clin Oncol 2004;22(9):1589-97.

[8] Scaglioti GV, Parikh P, von Pawel J, et al. Phase III study comparing cisplatin plus gemcitabine with cisplatin with pemetrexed in chemotherapy-naïve patients with advanced stage NSCLC. J Clin Oncol 2008;26(21):3543-51.

[9] Kris MG, Johnson BE, Kwiatkowski DJ, et al. Identification of driver mutations in tumour specimens from 1000 patients with lung adenocarcinoma; the NCI 's lung cancer mutation consortium (LCMC). J Clin Oncol 2011;29.

[10] Janne PA, Wang XF, Socinski MA, et al. Randomised phase II trial of erlotinib (E) alone or in combination with carboplatin/paclitaxel (CP) in never or light smokers with advanced lung adenocarcinoma: CALGB 30406. J Clin Oncol 2010;28:750a.

[11] Mok TS, Wu YL, Thongprasert S, et al. Gefitinib or carboplatin-paclitaxel in pulmonary adenocarcinoma. N Engl J Med 2009; 361:947-57.

[12] Lee JS, Park K, et al. A randomized phase III study of gefitinib (IRESSATM) versus standard chemotherapy (gemcitabin plus cisplatin ) as a first line treatment for never smokers with advanced or metastatic adenocarcinoma of the lung. JTO 2009; 4:283-4.

[13] NCCN Guidelines. Non small cell lung cancer, sensitizing EGFR mutation positive. Version 2.2015:17.

[14]Zhou C. First line therapy of advanced non small cell lung cancer with activating EGFR mutation. Lung cancer therapy annual. Informa Healthcare 2012(7); 7481.

[15] Gazdar AF. Activating and resistance mutations of EGFR in non-small-cell lung cancer: role in clinical response to EGFR tyrosine kinase inhibitors. Oncogene 2009; 28 (suppl 1): 24-31.

[16] Li D, Ambrogio L, Shimamura T, Kubo S, et al. BIBW2992, an irreversible EGFR/HER2 inhibitor highly eff ective in preclinical lung cancer models. Oncogene 2008; 27: 4702-11.

[17] Yang J, Wu Y, Schuler M, et al. Afatinib versus cisplatin-based chemotherapy for EGFRmutationpositive lung adenocarcinoma (LUX-Lung 3 and LUXLung 6): analysis of overall survival data from two randomised, phase 3 trials. Lancet Oncol. 2015;16(2):141-151. 


\section{International Journal of Science and Research (IJSR) \\ ISSN (Online): 2319-7064}

Index Copernicus Value (2015): 78.96 | Impact Factor (2015): 6.391

[18]LUX lung 7:A Randomised, Open-label Phase IIb Trial of Afatinib Versus Gefitinib as First-line Treatment of Patients With EGFR Mutation Positive Advanced Adenocarcinoma of the Lung. ClinicalTrials.gov NCT 01466660

[19] Mok T, Spigel DR, Park K, et al. Efficacy and safety of PF-00299804 (PF-299), an oral irreversible, pan human EGFR TKI , as first line treatment in selected patients with advanced NSCLC. J Clin Oncol 2010;28:7537a.

[20] Ou SH, Soo RA. Dacomitinib in lung cancer: a "lost generation" EGFR tyrosine-kinase inhibitor from a bygone era?. Dove Med Press 2015;9:5641-53. 